\title{
On the Characterization Results of Toroidal Transformer and Microtransformer Under Different Applied Voltages
}

\author{
F. Nascimento, A. C. C. Telles, and R. C. Teixeira \\ Renato Archer Center for Information Technology - CTI, Rod. D. Pedro I km 143.6 - Campinas /SP, Brazil \\ e-mails: fnascimento@cti.gov.br; antonio.telles@cti.gov.br; ricardo.teixeira@cti.gov.br
}

\section{Abstract}

This work is part of a study regarding an issue found in the characterization of a microtransformer when different voltage values were applied to measure the inductance and resistance values of the windings. Under such conditions it was found a variation on those parameters, for both primary and secondary sides.

In order to know if the mentioned issue was due to the reduced dimensions of the microtransformer, a transformer built using the same structure and geometry, but with dimensions much larger than those of the microtransformer, was also characterized for different voltage values. As a result, it was verified that the variation of inductance and resistance values are not related with the transformer dimensions.

\section{Introduction}

Over the past few decades, the size reduction of several electronic devices has been continuous and so that their performance is not compromised. Exceptions to this are inductors and transformers, which in order to have their size reduced, it was necessary to give up one of its main characteristics: the inductance values, which decrease in the same proportion as the dimensions are reduced.

Once a microtransformer is built, proper characterization is required in order to know its main physical parameters (inductances and resistances) as well as those related to performance (quality factors and coupling coefficients, for instance). In most works, the voltages applied on the devices to perform their characterization have values much lower than those in which they will operate in practice.

In tests carried out with a $4 \mathrm{~mm}$ toroidal microtransformer, that was built using wire bonding and Multi-Chip Module (MCM) technologies [1], variations in the measured inductance and resistance values, in more than one operating frequency, were identified when the measurements were performed applying different voltage values on the device's coils. This behavior was found previously in the work of Macrelli et al, for a microtransformer that is similar, in dimensions, structure and material, to that we are using [2].

Thus, in order to identify whether this was a problem related to the small dimensions of the microtransformer, the same procedure was executed with a toroidal transformer (which will only be called a transformer, from here), whose dimensions are much larger than those of the microtransformer, in order to check possible variations in inductance and/or resistance values by applying different voltage values on its windings.

\section{Experimental setup and procedure}

The dimensions and basic characteristics for each device are shown in Table 1. Both microtransformer and transformer have ferrite cores whose properties are described in [3] for the first and in [4] for the second one, and were assembled in solenoid structure and toroidal geometry.

All the inductance and resistance measurements were made using a LCR meter model E4980A from Keysight Technologies. Calibration and compensation procedures of the LCR meter were carried out prior to the data acquisition and each time the voltage level was adjusted. Both inductance and resistance measurements were made in the frequency range from $10 \mathrm{kHz}$ and 2 $\mathrm{MHz}$. The uncertainties on inductance and resistance measurements ( $\delta L$ and $\delta R$ ) were assumed to be the average errors found on measurements of inductance and resistance values of a standard inductor and resistor, being equal to $10 \mathrm{nH}$ and $1 \mathrm{~m} \Omega$, for $L$ and $R$, respectively. The LCR meter was configured to apply sinusoidal voltage signals.

\section{Results and discussion}

As can be seen in Figs. 1 and 2, the inductance curves as a function of frequency for primary and secondary windings of both devices, show variations in values and behaviour when subjected to different

Table I: Main features and dimensions of the magnetic devices.

\begin{tabular}{|c|c|c|c|c|c|c|c|c|c|c|}
\hline Device & Core material & $\mathrm{B}_{\text {Sat }}(\mathrm{T})$ & $\mathrm{H}_{\text {sat }}(\mathrm{A} / \mathrm{m})$ & $\mu_{\mathrm{i}}$ & Structure/Geometry & $\mathrm{d}_{\mathrm{ext}}(\mathrm{mm})$ & $\mathrm{d}_{\text {int }}(\mathrm{mm})$ & $\mathrm{h}(\mathrm{mm})$ & $\mathrm{N}_{1}$ & $\mathrm{~N}_{2}$ \\
\hline Microtransformer & Ferrite (N30) ${ }^{[3]}$ & 0.38 & 1200 & 4300 & Solenoid/Toroidal & $4.00 \pm 0.12$ & $2.40 \pm 0.12$ & 0.49 & 1 & 19 \\
\hline Transformer & Ferrite $^{[4]}$ & 0.5 & 2000 & 4500 & Solenoid/Toroidal & $72.2 \pm 0.5$ & $39.7 \pm 0.5$ & $52.0 \pm 0.5$ & 4 & 16 \\
\hline
\end{tabular}


voltages during the measurement procedures. From Figs. 3 and 4, it can be seen that the resistance values of the windings also present some variation under different voltage levels for both devices, but the effects on resistance values are not as pronounced as on the inductance ones.

From the presented data we can safely say that the variation in the values of the parameters is a problem that does not depend on the dimensions of the devices. Although, the microtransformer is much more affected than the transformer, because the amplitude of the voltage variation is much smaller in it but the differences in the inductance readings are proportionally greater than in the case of the transformer. The causes of the variation in the inductance values for different voltages applied to the windings are still being analysed. However, for the transformer, it is difficult to believe that it is related to the saturation of the core, because given the dimensions of such a device, a voltage of $5 \mathrm{~V}$ is not enough to generate an electric current and, consequently, a magnetic field flow intense enough to reach saturation in the measured frequency range, which occurs with a field of $\sim 0.5 \mathrm{~T}$ at $\sim 2 \mathrm{kA} / \mathrm{m}$ [4].

In the case of the microtransformer, another possible cause, but which may be unlikely, for the occurrence of different inductance values with the voltage variation is the uncertainty in the measurements, as these are in the order of $10 \mathrm{nH}$. However, the variations observed in the $L$ values are of the order of uncertainty in the

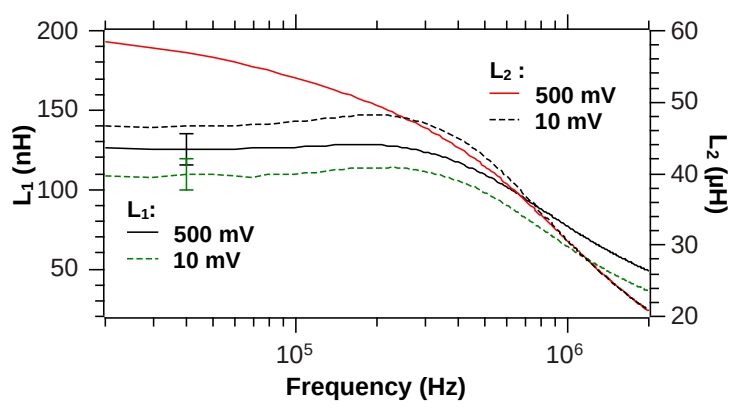

Fig.1. Inductances as a function of frequency for the microtransformer.

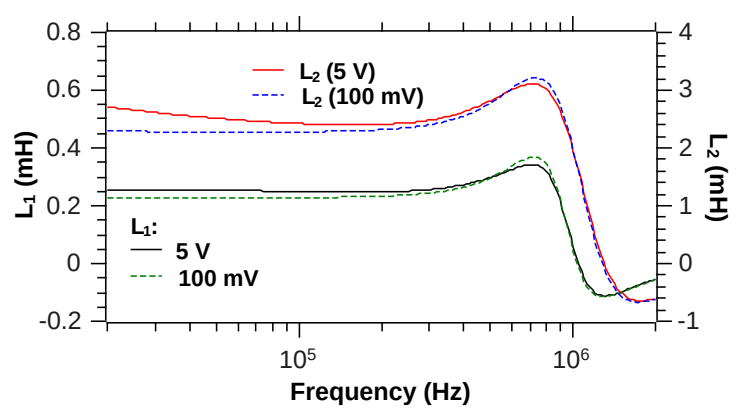

Fig.2. Inductances as a function of frequency for the transformer.

measurements only in the results obtained for the

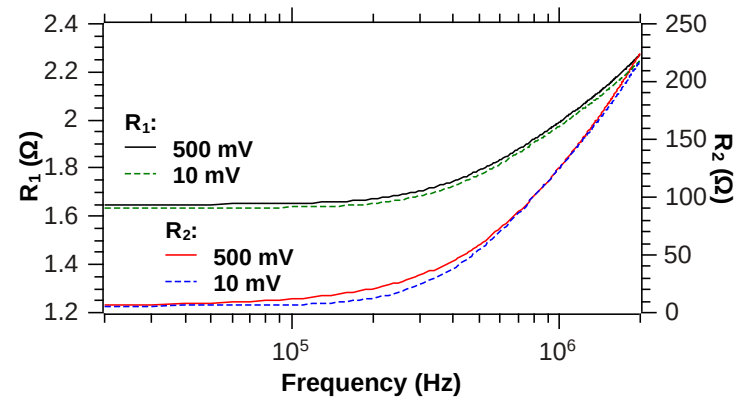

Fig.3. Resistances as a function of frequency for the microtransformer.

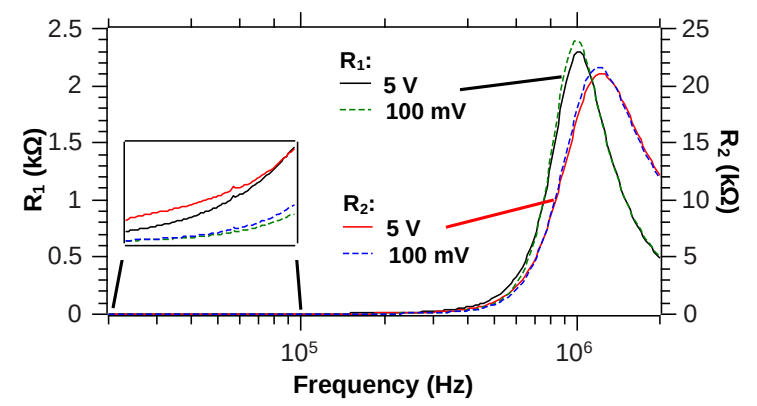

Fig.4. Resistances as a function of frequency for the transformer.

primary winding of the microtransformer, but are much greater in the results of some of the measurements made in the secondary winding. It is possible that the nonlinearity of the B-H curve may be one of the reasons for this to happen.

\section{Conclusions}

Based on the fact that both devices present variation in inductance and resistance values when subjected to different voltage levels, we can conclude that this issue is not a problem directly linked to the small dimensions of the microtransformer. Works in order to avoid or minimize this kind of problem are in progress.

\section{Acknowledgments}

The authors thank to M. M. Rocha who built the microtransformer prototype and to NAPE/CTI. This work was supported by CNPq, grant \#301621/2020-9.

\section{References}

[1] F. Nascimento et al., "A 4 mm toroidal microtransformer built with wire bonding and MCM technologies," in 2019 34th Symposium on Microelectronics Technology and Devices (SBMicro), pp. 1-4, August 2019

[2] E. Macrelli et al., "Modeling, design, and fabrication of highinductance bond wire microtransformers with toroidal ferrite core,” IEEE Trans. Power Electron., vol. 30, no. 10, pp. 57245737, Oct. 2015

[3] Datasheet for N30 material, series B64290P0036 - EPCOS AG 2017, May 2017

[4] R. F. Gribble, “A ferrite loaded piggy-back crowbar gap”, Proceedings of symposium on engineering problems of fusion research, LANL, DI-5-1 to DI-5-3, Los Alamos, N. M., 1969 